\title{
Estrategia didáctica de la medicina integrativa oriental y occidental en el manejo de la COVID-19
}

\section{Didactic strategy of eastern and western integrative medicine in the management of COVID-19}

\author{
Alejandro Bernardo Cervantes Palomino*1, Arlett María Iglesias Torres ${ }^{2}$, Rogelio Pérez Rivero ${ }^{1}$, Anabel Sarduy \\ Lugo $^{3}$ \\ ${ }^{1}$ Hospital Clínico Docente "Ernesto Guevara" de las Tunas, Cuba \\ ${ }^{2}$ Hospital Liborio Panchana Sotomayor, Ecuador \\ ${ }^{3}$ Universidad Estatal Península de Santa Elena, Ecuador \\ *cervapal@gmail.com
}

DOI: https://doi.org/10.26871/killkana_salud.v4i3.693

\begin{abstract}
Resumen
Contexto: La integración de la medicina tradicional china (oriental) y la medicina moderna (occidental) en el tratamiento del nuevo coronavirus SARS-CoV2 (Covid-19) constituye un desafío para el personal médico y de enfermería en el tratamiento y prevención de la COVID-19. Objetivo: establecer estrategias didácticas y de actuación en el tratamiento ante la COVID-19 mediante el empleo de la medicina natural y tradicional integrada a la medicina occidental. En el Hospital Provincial Dr. Ernesto Guevara de la Serna en la provincia de Las Tunas en el mes de mayo del año 2020. Metodología: se realizó un estudio de intervención dirigido a médicos y enfermeros que laboran en los diferentes servicios del Hospital Dr. Ernesto Guevara de la Serna en Las Tunas, Cuba en mayo de 2020, que atienden a pacientes diagnosticados o con sospecha de COVID- 19. Se seleccionaron criterios ya establecidos y normados por protocolos para tratar a estos pacientes. Resultados: se diseñó una estrategia didáctica para contribuir con la diversidad del personal médico y de enfermería que laboran en áreas destinadas a enfermos de COVID-19. La estrategia se estructuró a partir de un único objetivo general, además de acciones específicas, que partieron de la unificación de la medicina convencional (occidental) y la medicina natural y tradicional (oriental), las acciones destinadas a cada grupo de personas según el área de desempeño, así como su profesión y desempeño. Conclusiones: la estrategia didáctica propuesta favorece el desempeño profesional y la calidad de los servicios por parte del personal médico y de enfermería.
\end{abstract}

Palabras clave: coronavirus, estrategias, medicina convencional, medicina natural.

\begin{abstract}
Introduction: The integration of traditional Chinese (eastern) medicine and modern (western) medicine in the treatment of coronavirus (Covid-19) constitutes a challenge for medical and nursing personnel in the treatment and prevention of COVID-19. Objective: to establish didactic and action strategies in the treatment of COVID-19 using natural and traditional medicine integrated into western medicine. At the Provincial Hospital Dr. Ernesto Guevara de la Serna in the Province of Las Tunas in May 2020. Methods: an intervention study was conducted for doctors and nurses who work in the different services of the Hospital Dr. Ernesto Guevara de la Serna Las Tunas, Cuba in May 2020, serving patients diagnosed with or suspected of COVID-19. Criteria already established and regulated by protocols were selected to treat these patients. Results: a didactic strategy was designed to contribute to the diversity of medical and nursing personnel working in areas intended for COVID-19 patients. The strategy was structured around a single general objective, in addition to specific actions, which started from the unification of conventional medicine (western) and natural and traditional medicine (eastern), the actions aimed at each group of people according to the area of performance, as well as their profession and performance. Conclusions: the proposed didactic strategy favors professional performance and the quality of services by medical and nursing personnel.
\end{abstract}

Keywords: coronavirus; strategies; conventional medicine; natural medicine.

\section{Introducción}

Cuando en el mes de diciembre del 2019 las autoridades de la República Popular China informaron a la Organiza- ción Mundial de la Salud (OMS) varios casos de neumonía de etiología desconocida en Wuhan, ciudad situada en la provincia china de Hubei. Una semana más tarde confir- 
maron que se trataba de un nuevo coronavirus que ha sido denominado SARS-CoV-2. Al igual que otros de la familia de los coronavirus, este virus causa diversas manifestaciones clínicas englobadas bajo el término COVID-19, que incluyen cuadros respiratorios que varían desde el resfriado común hasta cuadros de neumonía grave con síndrome de distrés respiratorio, shock séptico y fallo multiorgánico. La mayoría de los casos de COVID-19 notificados hasta ese momento debutaban con cuadros leves. ${ }^{1}$

Considerada una pandemia, ha afectado a 216 países, territorios o áreas, con 6750521 casos confirmados y 395779 fallecidos. En nuestro país ascienden a 2191 confirmados y 83 fallecidos acumulados desde el 11 de marzo de 2020. Los datos corresponden a los existentes en el momento de redactar este artículo. ${ }^{2}$

La repercusión psicoemocional que conlleva a trabajar bajo circunstancias extremas implica un desmoronamiento en el sistema psico inmunológico por el pánico que engendra estas situaciones. De ahí la importancia de que tengamos claro qué es lo que vamos a hacer, siendo conscientes de los riesgos que implican el no cumplir con todas las normas sanitarias descritas ante tal situación. Igualmente es importante saberse seguros y a buen resguardo a nuestros seres queridos para cumplir con éxito esta noble tarea de salvar vidas. Lo que nos lleva considerar una nueva normalidad, en presencia de una COVID 19 que marcara sin dudas un antes y un después.

Las normas de un lavado de mano adecuado, el uso del nasobuco y cambio frecuente del mismo, utilizar los medios de protección con que contemos, no olvidar que en el traslado desde y hasta el hogar y o al trabajo podemos contagiarnos, por lo que debemos de estar muy alertas para no contaminar a nuestro entorno y conservar la salud de nuestros familiares al retornar a nuestros hogares.

Existen factores de riesgo que predisponen o favorecen la COVID-19:

\section{- $\quad$ Edad}

- Déficit inmunológico

- Hacinamiento

- Contaminación ambiental, humo del cigarro o tabaco (ya sea de forma pasiva o activa)

- Enfermedad de base HTA, Diabetes, Cardiopatías, Asma, entre otras.

En gran medida todos somos vulnerables ante esta pandemia. En países desarrollado como China, Italia, España, Francia o Estados Unidos, entre otros, ha quedado demostrado que no importa la edad, sexo, raza, credo o religión, jerarquía o nivel económico, todos, sin excepción, somos vulnerables. Los más disciplinados son los que más posibilidades tienen de sobrellevar mejor a la pandemia ante los menos disciplinados.

Un punto de detección importante de casos de COVID19 es la urgencia hospitalaria. Lograr realizar la triada correspondiente, tiene como objetivo identificar, priorizar y aislar al paciente grave. Al tratarse de una enfermedad transmisible se deben extremar las medidas de precaución. ${ }^{3}$
Para lograr una adecuada clasificación y calificación de los pacientes, el personal de enfermería debe observar y tomar los signos vitales. En los casos de COVID-19 la gravedad de estos puede variar e incluso es posible que, con frecuencia, no se pueda determinar hasta qué punto esté comprometida la salud del paciente. En la triada, la medición de los cuatro signos vitales tradicionales (temperatura, presión arterial, frecuencia cardiaca y frecuencia respiratoria) puede suponer un riesgo de transmisión para el personal de enfermería y médicos, así como para el público en general. Por ello el personal médico y paramédico debe elevar todas las medidas de precaución para evitar el contagio. $^{3}$

Cumpliendo lo planteado por la educación médica superior, desde el mes de marzo, fueron emitidas las indicaciones para la reorganización del proceso docente., ${ }^{4} \mathrm{~A}$ partir de lo antes señalado el autor considera un grupo de Orientaciones generales

Donde se recomienda.

1) Disponer de dispensadores, con solución hidroalcohólica, ofrecer nasobucos y sustitución de estos cuando estén húmedos.

2) Establecer dos zonas: una para diferenciar a los pacientes con enfermedades respiratorias y otra a los pacientes con otros motivos de visitas a consultas.

3) Se identificará al personal que va a trabajar con cada uno de los grupos, teniendo cada grupo de trabajo los equipos e instrumental independientes para evitar el contagio.

4) En el primer contacto, el paciente deberá ser identificado según el grupo que corresponda a medida que vaya llegando al hospital.

5) Establecer información visual (carteles, folletos, etc.) en lugares estratégicos para proporcionar a los pacientes las instrucciones sobre lavado de manos, higiene respiratoria y como toser o estornudar en la flexura de la articulación del codo.

\section{Material y Métodos}

Se realizó un estudio de intervención en el hospital provincial Dr. Ernesto Guevara de Las Tunas en el mes de mayo del año 2020 con el objetivo de crear una estrategia didáctica con el fin de integrar a la medicina occidental y oriental para tratar a pacientes con sospecha o pruebas diagnósticas positivas de COVID-19.

En el mismo se incluyeron a todo el personal de enfermería y medico quedado distribuido por 44 médicos especialistas y residentes de todas las especialidades que laboraron en esta tarea, también por 59 técnico y licenciados en enfermería

En caso de pacientes que requieran de aislamiento se mantendrá hasta transcurridos 14 días desde el inicio de los síntomas, siempre que el cuadro clínico se haya resuelto. El seguimiento y el alta serán supervisados por su médico de atención primaria o de la forma que sea establecida por las áreas de salud según orientación del MINSAP, las opciones 
terapéuticas integradas de ambas medicinas siempre que sean los pacientes tributarios serán indicadas.

La vigilancia e investigación de casos y de los contactos son fundamentales para definir e identificar los cambios en la epidemiología de las infecciones humanas y seguirán brindando recomendaciones sobre control de infección. Se harán modificaciones a estas indicaciones, según sea necesario, a medida que se disponga de información adicional.

Se realizara desinfección con el uso del jabón ozonizado para el lavado frecuente de las manos será imprescindible para evitar el contagio, que se realizara: Antes de iniciar las labores, antes de realizar procedimientos invasivos, antes y después de atender pacientes, se usen guantes o no, antes y después de manipular heridas, después de estar en contacto con líquidos de Precaución dispuestos aunque se estén usando guantes, antes y después de entrar en cuartos de aislamiento, Durante la atención de pacientes, al moverse de un sitio contaminado a uno no contaminado del cuerpo del paciente, después del contacto con objetos inanimados en los alrededores inmediatos del paciente, Antes de colocarse y después de retirarse los equipo de protección personal (EPP), antes de ponerse guantes e inmediatamente después de quitárselos, después de usar el baño.

\subsection{Tratamiento según clasificación de hospitales}

Las políticas de los procedimientos con pacientes y fortalecimiento de la estructura de control de infecciones habitual, la ventilación adecuada, la ubicación correcta de los pacientes e higiene del entorno pueden reducir la diseminación de algunos patógenos respiratorios en los establecimientos de salud.

Evitar contacto de piel o mucosas con sangre y otros líquidos. Aunque se estén usando guantes, lavar las manos antes y después de entrar en cuartos de aislamiento. Durante la atención de pacientes, al moverse de un sitio contaminado a uno no contaminado del cuerpo del paciente. Después del contacto con objetos inanimados en los alrededores inmediatos del paciente. Antes de colocarse y después de retirarse los EPP. Antes de ponerse guantes e inmediatamente después de quitárselos. Después de usar el baño.

Si no existiera un sistema de suministro de aire independiente, deben apagarse los aires acondicionados (climatizadores) y abrir las ventanas para garantizar una buena ventilación. Asegurarse que las ventanas abren hacia lugares alejados del público.

- Siempre que sea posible, debe utilizarse equipo desechable en la atención de los pacientes afectados por nuevo coronavirus 2019

- Evitar procedimientos inductores de tos y esputo (aerosoles, broncoscopía, etc.)

- Todo el personal con acceso a la unidad de aislamiento debe usar Equipos de Protección Personal (EPP). El personal con inmunocompromiso no debe realizar esta actividad

\section{Resultados}

En el presente trabajo el autor recomienda la utilización de la terapia medicamentosa descrita por el protocolo nacional de salud como terapia occidental y las técnicas de la medicina oriental, también descritas y aprobadas por el ministerio de salud pública, a pesar de que hasta el momento no está identificada una droga antiviral totalmente eficaz, ni una vacuna, para la COVID 19 se propone la integración de estas terapias que se aplicara por los 44 médicos y los 59 enfermeros.

A continuación, se expone la estrategia didáctica de actuación con pacientes sospechosos o portadores de la COVID19. Por el personal médico y de enfermería.

Objetivo general: proponer una estrategia didáctica ante pacientes sospechosos o portadores de la COVID 19 para médicos y personal de enfermería.

El tratamiento médico medicamentos se realizara según protocolo de actuación, donde se recomienda el siguiente tratamiento en pacientes hospitalizados:

- Kaletra (200 Lopinavir -50 Ritonavir) 2 cápsulas cada 12 horas por 30 días.

- Cloroquina (250 mg = $150 \mathrm{mg}$ base) 1 tableta cada 12 horas por 10 días.

En la paciente obstétrica mayor de 17 años con 50 o más kilogramos de peso emplear $500 \mathrm{mg}$ dos veces al día por 10 días. En personas de menos de $50 \mathrm{~kg}$ emplear esa dosis los dos primeros días y luego $500 \mathrm{mg}$ una vez al día los siguientes ocho días.

De no existir contraindicaciones ni signos de gravedad, para su administración, Interferón alfa 2 b (3 millones de unidades, por vía intramuscular, 3 veces a la semana por cuatro semanas. Evaluar la evolución de la enfermedad y determinar continuidad de este tratamiento.

- Antibióticos de amplio espectro si sospecha sobreinfección bacteriana.

- Tratamiento de las comorbilidades, de acuerdo a su estado de compensación.

\section{Actuación de enfermería}

La actuación de enfermería se hará de una forma precisa e inmediata dependiendo del estado del paciente y de las indicaciones médicas se podrán utilizar algunas de las variantes de la medicina tradicional descritas e indicadas, $o$ como acción independiente de enfermería. Pero sin dejar de señalar los elementos que el enfermero considere destacar. Desde la llega del paciente y las indicaciones médicas las que deberán de cumplirse desde la llegada dl paciente a la sala.

\subsection{Tratamiento Naturista}

Tratamiento de enfermos:

[En pacientes con síntomas leves o manifestaciones generales, utilizar dos o tres puntos de los grupos (1) y (2), en casos graves dos o tres puntos del grupo (3)]:

1) Hegu (IG-4), Taichong (H-3), Tiantu (Ren-22), Chize (P-5), Kongzui (P-6), Zusanli (E-36), Sanyinjiao (Bp$6)$, 
2) Dazhu (V-11), Fengmen (V-12), Feishu (V-13) Xinshu (V-15), Geshu (V-17),

3) Zhongfu (P-1), Danzhong (Ren- 17), Qihai (Ren-6), Guanyuan (Ren-4), Zhongwan (Ren-12)

Según síntomas se podrán utilizar los siguientes puntos:

- Fiebre persistente: Dazhui (Du-14), Quchi (IG-11),

- Opresión en el pecho, dificultad para respirar: Neiguan (Pc-6), Lieque (P-7), Fenglong (E-40), Dingchuan (EXT-1),

- Diarreas, heces blandas: Tianshu (E-25), Fenlong (E40),

- Febrícula o afebril, vómitos, heces blandas: Feishu (V13), Tianshu (E-25), Fujie (B-14), Neiguan (Pc-6).

\subsection{Unidad de cuidados intensivos}

\subsubsection{Equipamiento y ambiente}

- Si es posible, usar equipamiento desechable

- Asigne equipamientos, como estetoscopios, brazalete de presión sanguínea, termómetros, etc., para individualizar a los pacientes a los que se aplican las Precauciones de Contactos.

- $\quad \mathrm{Si}$ es necesario compartir el equipamiento entre pacientes, se lo debe limpiar y desinfectar después del uso con cada paciente.

- Los trabajadores de la salud deben evitar tocarse los ojos, la nariz, o la boca con sus manos con o sin guantes ya que podrían estar potencialmente contaminadas.

- Evite contaminar las superficies ambientales que no están directamente relacionadas con la atención de los pacientes (por ej., manijas de puertas, interruptores de luz).

Ubicación de los pacientes: usar habitaciones individuales o formar cohortes de pacientes con el mismo diagnóstico etiológico puede facilitar la aplicación de medidas de control de infección.

Equipo Protección Personal: Al ingresar a la sala / al área de aislamiento o al atender a un paciente con una infección transmitida por aire obligada / preferencial en otros entornos, use un respirador para partículas que alcance como mínimo el nivel de protección de un N95 o equivalente.

\subsubsection{Ubicación de pacientes}

- Coloque al paciente en una sala de prevención para la transmisión aérea (es una "sala de prevención de la transmisión aérea" una habitación con $\geq 12$ ACH (12 o más intercambios de aire total por hora) y dirección de flujo de aire deseada, la cual puede lograrse con ventilación mecánica o natural.

- $\quad$ Si no hay disponibilidad de una sala de aislamiento ventilada, ubique a los pacientes en habitaciones separadas bien ventiladas.

- $\quad$ Si no se dispone de habitaciones individuales, forme cohortes de pacientes de acuerdo con el mismo diagnóstico etiológico en lugares bien ventilados.
- Los procedimientos que generan aerosoles con transmisión de patógenos deben realizarse usando el Equipo Protección Personal apropiado en una sala de prevención de la transmisión aérea.

\subsubsection{Equipo Protección Personal}

- Como mínimo, use un naso buco médico (quirúrgica o naso buco de procedimientos) bien ajustada, al ingresar a la habitación del paciente; el uso de máscara es obligatorio si se trabaja a $\leq 1 \mathrm{~m}$ del paciente.

- Cuando realice procedimientos que generan aerosoles asociados con transmisión de patógenos, use un respirador para partículas que alcance como mínimo un nivel de protección de un N95 certificado o equivalente, guantes, bata y protección ocular (gafas protectoras).

Recepción de enfermería

La recepción de enfermería se hará de una forma clara y breve pero sin dejar de señalar los elementos que el enfermero considere destacar, orientación, conciencia, pupila y motilidad. Desde la llega del paciente y la primera impresión del profesional al ver llegar al paciente como llega su estado de concentración, si trae o no puesto el nasobuco, si es torpe al deambular y si necesita de apoyo para trasladarse, si están protegidas las personas que lo acompañan enfatizar en las deposiciones si ha tenido diarrea y en la orina

Hasta el momento no está identificada una droga antiviral totalmente eficaz, ni una vacuna no obstante se recomienda:

- Kaletra (200 Lopinavir -50 Ritonavir) 2 cápsulas cada 12 horas por 30 días.

- Cloroquina (250 mg = $150 \mathrm{mg}$ base) 1 tableta cada 12 horas por 10 días.

- Mantener Interferón A2B: 3 millones de unidades IM días alternos por un mes.

- Ceftriaxona 1 gr a 2 gr cada 12 horas: en pacientes donde se diagnóstica infección bacteriana asociada.

- La decisión de utilización de otro antibiótico estará determinada por los resultados de estudios de laboratorio y el mapa microbiológico del servicio.

- Uso del Ac Monoclonal Itolizumab (bbo $25 \mathrm{mg}$ ) en dosis de $200 \mathrm{mg}$ ( 8 bbos) en $200 \mathrm{ml}$ de Solución salina a durar 2 horas. La dosis puede ser hasta $400 \mathrm{mg}$ si fuera necesario subir dosis para obtener mayor efecto clínico. Estas dosis estarían en los rangos 2,9 mg por kilo de peso y $5,7 \mathrm{~kg}$ de peso, respectivamente. La misma dosis puede ser repetida a los 5-7 días de la dosis inicial, según respuesta.

\subsubsection{Otras alternativas terapéuticas}

- Surfacen. (instilación por tubos orotraqueal a la dosis recomendada) en pacientes con SDRA. Ver preparación en formulario nacional. 100mg instilados cada 8 horas. Comenzar al intubar precozmente. Sedación y relajación muscular 


\subsubsection{Mantenimiento de sedación adecuada}

- Emplear Midazolam (ampollas de $10 \mathrm{mg} / 2 \mathrm{ml}$ ). La sedación puede ser con bolos de 1-2 mg I.V. repitiendo la dosis hasta el nivel deseado, también Diazepam a 10 mg en bolo, no se aconseja la infusión de Midazolam por la ocurrencia de delirio. La relajación se empleará en casos ventilados que mantengan asincronías con el ventilador que no respondan a esquemas de sedación según los protocolos.

- Alternativa el Propofol con la precaución de vigilancia hemodinámica, a nivel de sedación, bolo 1-1,5 mg x kg, iniciar mantenimiento $0,3 \mathrm{mg} \times \mathrm{kg}$ x hora, se aumenta en 0,3 cada 10 min solo para mantener el nivel de sedación.

- Relajación muscular: Debe evitarse. De ser necesaria se usarán los relajantes musculares no despolarizantes, con preferencia el Atracurio o el Vecuronio y en menor medida el Pancuronio. Usarla el menor tiempo posible, nunca por un periodo mayor de 48 horas.

- VECURONIO: Bolo IV $4 \mathrm{mg}(0,07-0,10 \mathrm{mg} / \mathrm{kg})$. Infusión $3-10 \mathrm{mg} / \mathrm{h}(0,02-0,05 \mathrm{mg} / \mathrm{kg} / \mathrm{h})$

- ATRACURIO: bolo 20-30 mg (0.4-0,5 mg/kg). Infusión $20-25 \mathrm{mg} / \mathrm{h}(0,2-0,4 \mathrm{mg} / \mathrm{kg} / \mathrm{h})$

- PANCURONIO: solo en bolos $4 \mathrm{mg}(0,06-0,08 \mathrm{mg} / \mathrm{kg})$

\subsubsection{Los pacientes que no estén intubados y sean tribu-} tarios de la medicina natural

Acupuntura: Dar estimulación media cada dos días en los puntos V13, VG14, P6, E36. Si Hemoptisis: V17, P1, Si tos: P9; Si sudoración fría: C6; Si palpitaciones: C7; Si anorexia: B4, Vc12; Si diarreas: E25; Si trastornos genitales: R3, B6.

Moxibustion: Aplicar una vez al día de siete a diez moxas del tamaño de un grano de arroz en los puntos V13, V43. Se cauterizan durante 7 días y después de descansar 7 se repite el k.o. varias veces. También se puede cauterizar en los puntos V13, PFM 15 ( SaJwa), V17, V19 de la misma forma explicada anteriormente.

Fármacopuntura: Inyectar el medicamento en los puntos mencionados diariamente a lo largo de 20 días y descansar 10, es decir un ciclo de k.o. de 20 días inyectables y 10 de descanso. También se pueden utilizar otros puntos como V16, P1, Ig11 y P6.

\subsection{Convalecencia}

En la etapa de convalecencia se recomienda el uso de productos naturales como Nutrisol, Moringa en sus diferentes presentaciones, ViproloVimang extracto acuoso concentrado, con el objetivo de contribuir a la recuperación del organismo.

\subsubsection{Homeopatía}

En el tratamiento se propone el empleo del complejo homeopático ABAS (Arsenicum album30CH, Bryoniaalba
30CH, Aconitum napellus30CH y Spongiatosta $30 \mathrm{CH}$ ) para eltratamientosintómático de los pacientes. Se recomienda su empleo a razón de 5 gotas sublinguales tres veces al día, pudiendo incrementarse la frecuencia en correspondencia con las características del cuadro clínico en cada caso individual.

\subsubsection{Fitoterapia}

Caña Santa extracto fluido, vía oral, 20 gotas en medio vaso de agua 2 veces al día.

Antivirales. Ajo Tintura 20\%: V/O 20 gotas en $\frac{1}{2}$ vaso de agua $3 / v$ día.

Contraindicaciones: No tomar niños, Embarazadas ni mujeres que estén lactando. Advertencias: Puede producir trastornos digestivos y/o renales.

Propóleos Tintura 10\%: V/O de 3 a 15 gotas en $\frac{1}{2}$ vaso de agua se debe de ir aumentando gradualmente.

Manzanilla Tintura $20 \%$ V/O 20 gotas en un vaso de agua 3 veces al día.

Jengibre tintura $50 \% 10$ a 20 gotas en un vaso de agua 3 veces por día.

\subsubsection{Acupuntura}

E36, IG4, IG11, P1, VC17, VG20, VG14, P5, P7

(Aplicar moxibustion directa incompleta en VG14, E36, IG4, IG11.

\subsubsection{Otros tratamientos}

Insertar agujas en los puntos TF17, E9, VC22, VG14, V12 y P5 y dejar aplicada durante 20 o 30 min. Estimular una vez cada 5 min. Por giro.

Puntos principales: Neiguan(Pc-6), Zusanli (E-36), Zhongwan (Ren-12), Tianshu (E-25), Qihai (Ren-6).

\subsection{Otras Alternativas Naturistas}

CORTAFUEGOS INTERNO CONTRA EL CORONAVIRUS Medicina Tradicional China (MTC)

Este método se basa en 2000 años de la efectiva (MTC). A través del sistema de acupuntura activamos diversos puntos que les dan un fuerte empuje a nuestras defensas y crean unos cortafuegos internos para nuestro sistema respiratorio.

Si usamos este método todos los días nuestro cuerpo formará unos cortafuegos internos contra el virus. El método puede parecer muy simple y las substancias fáciles de conseguir, pero esto no nos debe llevar a engaño. El método puede activar muy efectivamente nuestras defensas y fortalecer y proteger nuestro sistema respiratorio, especialmente los pulmones, que son los que el coronavirus más pone en peligro.

IMPORTANTE: La mejor protección es no entrar en contacto con el virus. Hay que protegerse contra él. No seamos ingenuos ni descuidados. Debemos evitar aglomeraciones y grupos, así como mantener la distancia con otras personas. Es importante que nos desinfectemos y nos lavemos las manos regularmente. No nos toquemos la cara 
(nariz, ojos y boca). Debemos protegernos con máscaras y ventilar con frecuencia las habitaciones.

PRINCIPIO DEL MÉTODO: Estimular durante 810 horas al día puntos de acupuntura específicos, que actúan expresamente contra el virus, contra la fiebre y las enfermedades respiratorias, mejoramos nuestras defensas fortalecemos los pulmones y nuestra fuerza interna.

Puntos de acupuntura los parches con determinadas substancias colocar parches de esparadrapo con bálsamo chino puede estimular el sistema inmunológico.

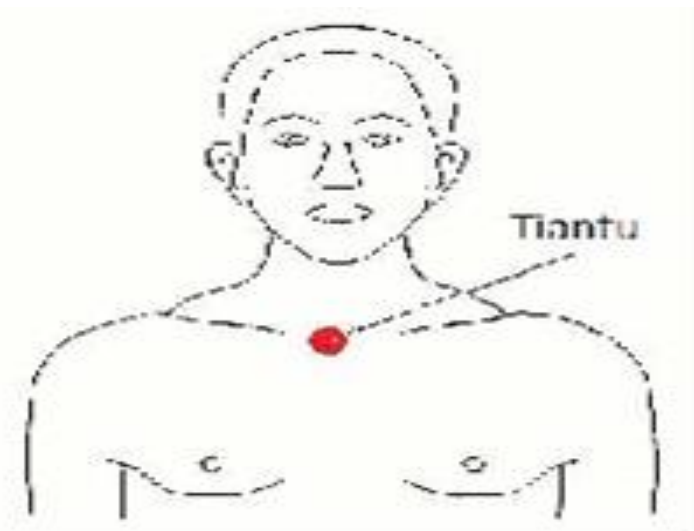

RenMai 22 (Vaso Concepción 22) TianTu天突

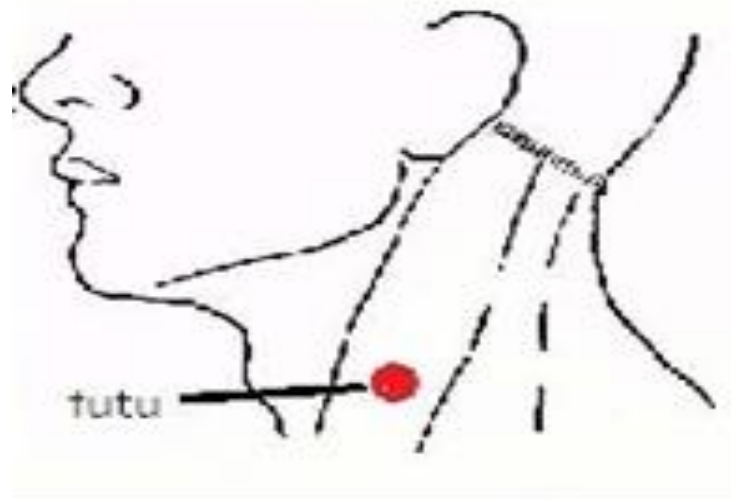

Intestino Grueso 18, FuTu扶突

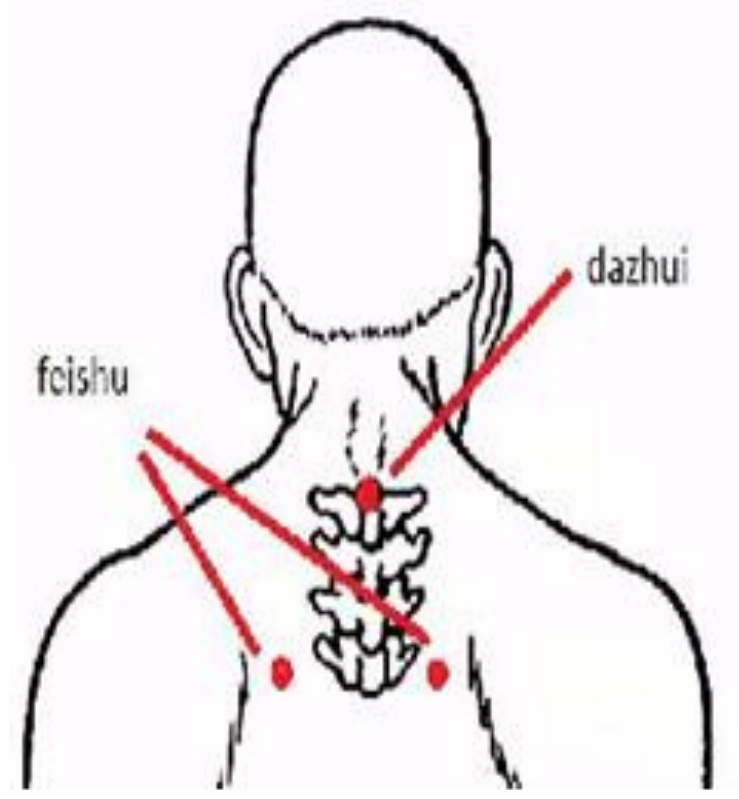

DuMai 14 (Vaso Gobernador 14, DaZhui 大椎

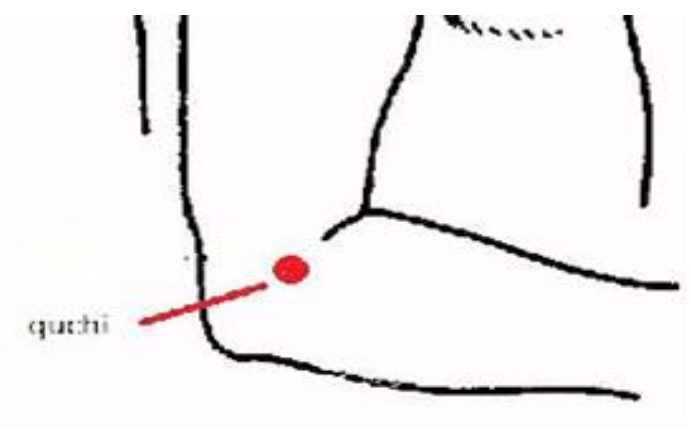

Vejiga 13, FeiShu. Intestino Grueso 11, QuChi曲池

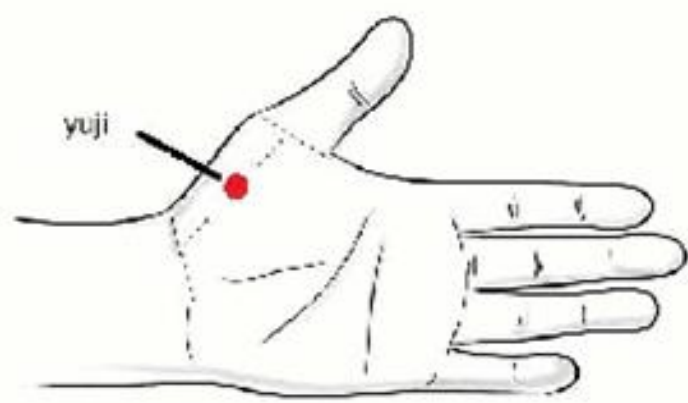

Pulmón 10, YuJi鱼际 


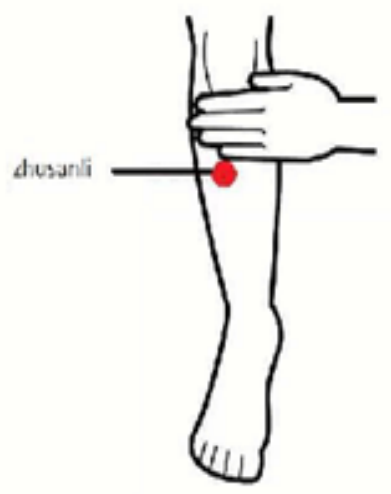

Estómago 36, ZhuSanLi, 足三里

\subsection{Digitopuntura}

Sistema inmunitario. Mejora CONORAVIRUS COVID19 la estimulación con digitopuntura en estos puntos con las técnicas apropiadas puede favorecer el sistema inmunológico.

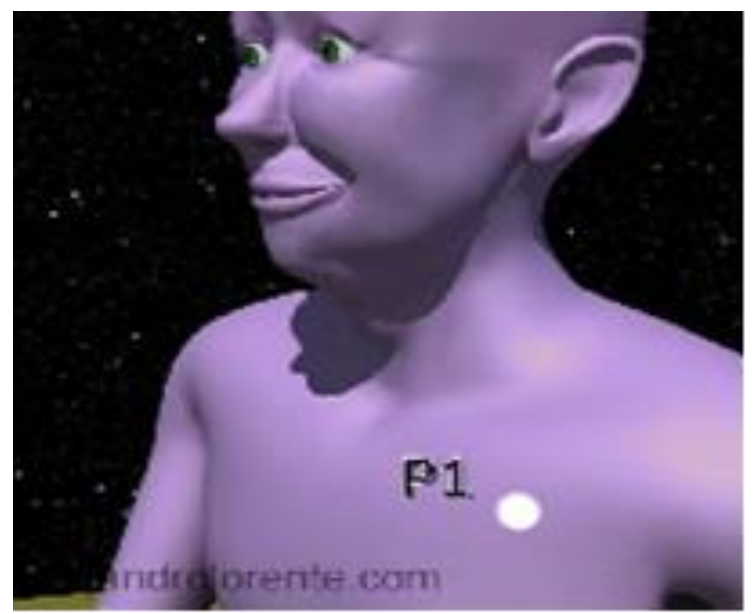

LOCALIZACIÓN: A $6 \mathrm{~cm}$ de la línea central del cuerpo, $1 \mathrm{~cm}$ por debajo de la clavícula

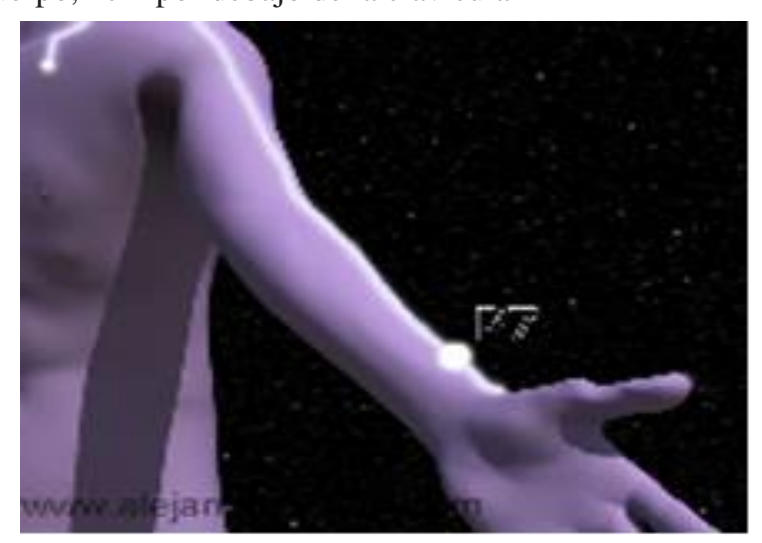

LOCALIZACIÓN: Colocando el dedo índice de una mano sobre el pulgar de la otra llegamos al punto exacto
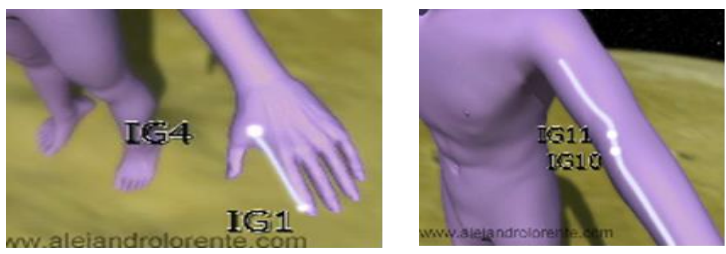

LOCALIZACIÓN: En una montañita situada entre el dedo gordo y el índice HORA ALGIDA 05-07

LOCALIZACIÓN: Interior del codo HORA ALGIDA 05-07

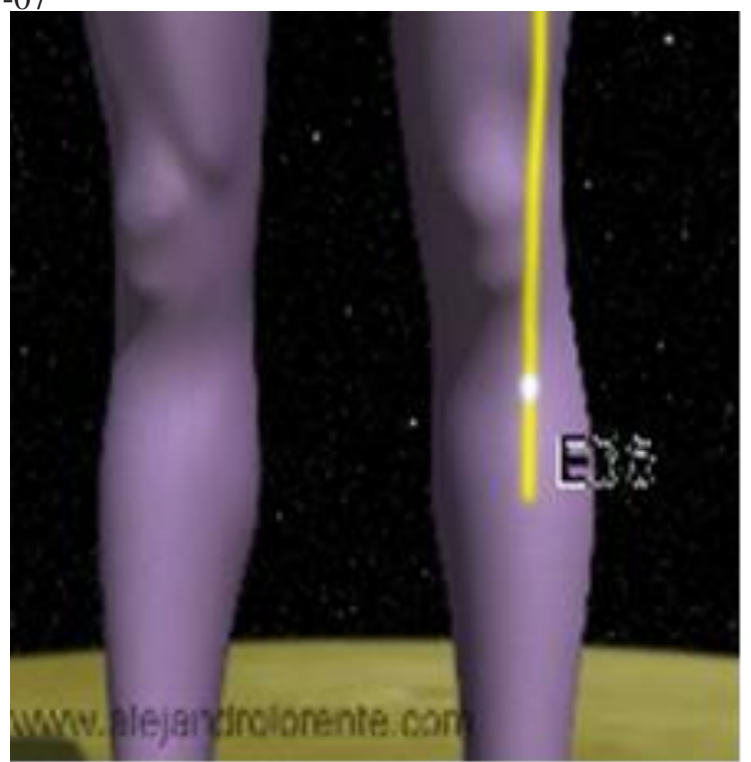

LOCALIZACIÓN: A $3 \mathrm{~cm}$ de la rodilla doblada HORA ALGIDA 07-09
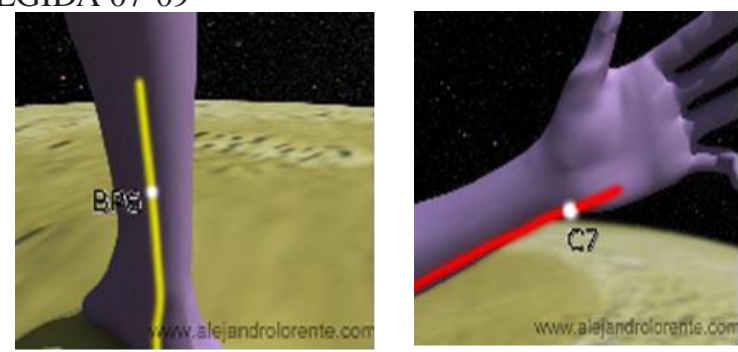

LOCALIZACIÓN: A $3 \mathrm{~cm}$ del borde superior del maléolo interno, en el borde trasero de la tibia. HORA ALGIDA 09-11

LOCALIZACIÓN: En la cara interna de la muñeca, en el borde que lleva al dedo meñique. HORA ALGIDA 11-13

\section{Fuente de Financiamiento}

Este estudio es autofinanciado.

\section{Conflicto de Intereses}

No existen conflictos personales, profesionales, financieroso de otro tipo.

\section{Consentimiento Informado}

Los autores cuentan con el consentimiento informado de los pacientes para la investigación, la publicación del caso y sus imágenes. 


\section{Referencias Bibliográficas}

1. ENTREVISTA «La combinación de la medicina tradicional china y occidental en el tratamiento del coronavirus (Covid19) ha demostrado ser efectiva" Por @FemeninoRural Publicado el Abr 1, 2020) - BEIJING, Feb. 18, 2020 (Xinhua) - A pharmacist weighs Chinese herbal medicines for patients infected with the novel coronavirus at Anhui Provincial Hospital of Traditional Chinese Medicine in Hefei, east China's Anhui Province, Feb. 18, 2020. (Xinhua/Bai Bin)

2. Covid19cubadata.uh.cu. [citado 8 de junio 2020]. Disponible en: https://www.cusobu.nat.cu/covid/\#cuba.

3. V: 2.1.- PROTOCOLO DE ACTUACIÓN NACIONAL PARA LA COVID -19 Manejo de los casos:

4. Ministerio de Salud Pública. Resolución No. 82/2020. Gaceta Oficial [Internet]. 2020 [citado 8 de junio 2020]; CXVIII (19): [aprox. 4p]. Disponible en: https://www.gacetaoficial.gob.cu/sites/default/files/goc2020-ex19_0.pdf.

5. Acciones educativas para atender la diversidad estudiantil en Estomatología en el contexto de la COVID-19 Taimí Santos-Velázquez, Sara Elena Panizo-Bruzón, Universidad de Ciencias Médicas de Las Tunas. Dirección de Ciencia e Innovación Tecnológica. Las Tunas. Universidad de Ciencias Médicas de Las Tunas. Facultad de Ciencias Médicas "Dr. Zoilo Enrique Marinello Vidaurreta". Las Tunas, Cuba. Correspondencia a: Taimí Santos-Velázquez, correo electrónico: taimisantos@1tu.sld.cu Recibido: 1 de junio de 2020 Aprobado: 9 de mayo de 2020 Revista Electrónica Dr. Zoilo E. Marinello Vidaurreta Vol. 45, número 4 ISSN 1029-3027 I RNPS 1824 julio-agosto 2020

6. la vuelta al mundo en 40 puntos Alejandro Lorente https: //youtu.be_ad9_BZ9_m4

7. Manejo en urgencias del COVID-19 Versión de 27 de marzo de 2020. Centro de Coordinación de Alertas y Emergencias Sanitarias. Dirección General de Salud Pública,
Calidad e Innovación. Sociedad Española de Medicina de Urgencias y Emergencias (SEMES). Juan González del Castillo. Fernando Ayuso Baptista. Guillermina Bejarano Redondo. Francisco Javier Candel. Miguel Garvi García. Esther Gorjón Peramato. Agustín Julián Jiménez. Ferrán Llopis Roca. Cristina López Paredes. Alejandro Martín Quirós. Mar Ortega Romero. Belén Rodríguez Miranda. Martín Ruiz Grinspan. Raúl Sánchez Bermejo. Rodrigo Sanz Lorente. Beatriz Valle Borrego. Andrés von Wernitz Teleki. Juan González del Castillo. Ana Pernía Sánchez.Sociedad Española de Medicina Interna (SEMI). Francisco Epelde Gonzalo.Consejo General de Colegios Oficiales de Médicos (CGCOM). Juan Martínez Hernández.Consejo General de Enfermería (CGE). Coordinación. Guadalupe Fontán Vinagre.

8. World Health Organizaiton. Novel Coronavirus (2019-nCoV) technical guidance. Disponible en: https://www.who.int/emergencies/diseases/novelcoronavirus-2019/technical-guidance

9. https://www.mscbs.gob.es/profesionales/saludPublica/ ccayes/alertasActual/nCov-China/

Recibido: 16 de febrero de 2020

Aceptado: 5 de septiembre de 2020 